\section{C0710 IS THERE ANY ANATOMIC BASIS FOR OFF LABEL USE OF CORONARY ARTERY STENT IN RENAL ARTERY IN SWINE?}

doi:10.1136/hrt.2010.208967.710

Zheng Bin, Yan Hongbing. Beijing Anzhen Hospital

Objective Coronary drug-eluting stents have been implanted in small renal arteries $(<5.0 \mathrm{~mm})$ to reduce restenosis. The aim of this study is to investigate the anatomic basis for off label use of coronary stent in renal artery in swine.

Methods Nine animals were examined in this study. Paired segments of coronary and renal artery were sampled from the same animal. Tunica media circumference referred to length of demarcation between tunica media and adventitia. Area of intima and tunica media was the area between intima circumference and tunica media circumference. Wall thickness (from intima to tunica media) was calculated as mean of four measurement in different directions. Inner diameter was calculated as intima circumference $/ \pi$. Corrected wall thickness was calculated as wall thickness/inner diameter $\times 100$.

Results Intima circumference and inner diameter were not significantly different between coronary and renal arteries. Tunica media circumference $(7.00 \pm 0.73 \mathrm{~mm}$ vs $8.30 \pm 0.48 \mathrm{~mm})$, wall thickness $(0.34 \pm 0.05 \mathrm{~mm}$ vs $0.50 \pm 0.11 \mathrm{~mm})$, area of intima and tunica media $\left(2.09 \pm 0.41 \mathrm{~mm}^{2}\right.$ vs $\left.3.38 \pm 0.73 \mathrm{~mm}^{2}\right)$, and corrected wall thickness $(21.4 \pm 1.96$ vs $32.0 \pm 9.70)$ were significantly less in coronary arteries than renal arteries.

Conclusion It seems that muscle layer in renal artery is stronger than coronary artery. Renal artery needs stronger radial force than coronary artery. The off label use of coronary stent in renal artery might be inappropriate.

\section{e0711 LONG TERM FOLLOW-UP OF PATIENTS WITH SIROLIMUS ELUTING STENT IMPLANTATION IN SMALL DIAMETER RENAL ARTERY}

doi:10.1136/hrt.2010.208967.711

Zheng Bin, Yan Hongbing. Beijing Anzhen Hospital

Objective To investigate the safety and efficacy of sirolimus eluting stent implantation in small diameter renal artery.

Methods 32 sirolimus eluting coronary stent $(3.0 \sim 4.0 \mathrm{~mm})$ were implanted in 26 patients with hypertension. Patients were followed for a median of 12 months with measurements of blood pressure, serum urea nitrogen and serum creatinine, duplex sonography and intra-arterial angiography.

Results Initial technical success was achieved in all patients. Renal artery dissection occurred in one patients after stent implantation. During follow up, amelioration of blood pressure was achieved in six patients $(23.1 \%)$, amelioration of renal dysfunction was achieved in three patients $(11.5 \%)$, deterioration of renal dysfunction occurred in three patients $(11.5 \%)$. In-stent restenosis occurred in two patients, one found by angiography at the 10th month after stent implantation, another by duplex sonography at the 16th month after stent implantation.

Conclusion Implantation of sirolimus eluting coronary stents in small diameter renal artery is technically feasible with efficacy and safety. Although there was no in-stent restenosis during the first 6 months after stent implantation, in-stent restenosis could occur later. Patients with sirolimus eluting coronary stent implantation in small diameter renal artery should be followed for more than one year. e0712 ACTINOBACILLUS ACTINOMYCETEMCOMITANS INFECTION
EXACERBATES MYOCARDIAL ISCHAEMIA-REPERFUSION INJURY IN MICE

doi:10.1136/hrt.2010.208967.712

${ }^{1}$ Wang Dongqing, ${ }^{2}$ Suzuki Jun-ichi, ${ }^{2}$ Ogawa Masahito, ${ }^{3}$ Hanatani Tomoya, ${ }^{3}$ Izumi Yuichi, ${ }^{3}$ Isobe Mitsuaki. ${ }^{1}$ Beijing Stomatological Hospital Capital Medical University; ${ }^{2}$ Tokyo University; ${ }^{3}$ Tokyo Medical and Dental University

Background Recent epidemiologic evidence suggests that periodontal infection may increase the risk of atherosclerosis and acute thromboembolic events. The present study investigated the contribution of the major periodontal pathogen, Actinobacillus actinomycetemcomitans (A.a.), on myocardial inflammation and injury after ischaemia-reperfusion (I/R).

Methods and Results Male C57Bl/6J mice were subjected to $30 \mathrm{~min}$ of coronary ligation, followed by 7 days of reperfusion. Either live A.a. $\left(10^{8} \mathrm{CFU}\right)$ or saline was administered intravenously for 2 days before surgery. A.a.-challenged mice showed significantly larger infarction $(53 \% \pm 6 \%)$ compared with control mice $(36 \% \pm 3 \%)$ $(p<0.05)$, given similar areas at risk $(64 \% \pm 6 \%$; vs $63 \% \pm 2 \%)$. A.a.inoculated mice also exerted more interstitial fibrosis.

Conclusion Taken together, these data demonstrate that systemic challenge with A. Actinomycetemcomitans, a major periodontal pathogen, may exacerbate myocardial ischaemia/reperfusion injury.

\section{e0713 EVALUATING SUCCESSFUL ABLATION OF SCAR-RELATED ATRIAL TACHYCARDIA ORIGINATING AT LATERAL WALL OF RIGHT ATRIUM WITH A NEW METHOD: STRATEGIC LINEAR ABLATION TO SCAR AREA ISOLATION}

doi:10.1136/hrt.2010.208967.713

${ }^{1} \mathrm{Hu}$ Jianqiang, ${ }^{2}$ Wang Shengqiang, ${ }^{1}$ Cao Jiang, ${ }^{1}$ Oin Yongwen, ${ }^{1}$ Huang Xinmiao, ${ }^{1}$ Zhou Bingyan. ' Changhai Hospital; ${ }^{2}$ Pla 148th Hospital

Background Scar-related Intra-atrial re-entrant tachycardias (IARTs) located at lateral wall of right atrium are common late after cardiac surgery in which right lateral atriotomy was performed, and also occurred in some patients without prior atriotomy. Conventional mapping and ablation is relatively difficult because of the complicated anatomy and multiple potential re-entry loops.

Objective The study aims to investigate a new method of strategic linear ablation to isolation of scar area from right lateral wall for successful ablation of scar-related atrial tachycardia originating at lateral wall of right atrium.

Methods Four patients had AT related to myocardial scar or incision located at lateral wall of right atrium underwent the electrophysiological study and RF catheter ablation. Earliest activation combined with entrainment mapping was adopted to determine a critical isthmus. Scar area isolation from right lateral wall was performed by linear ablation along cavotricuspid isthmus, from the scar to crista terminalis and to the inferior vena cava (IVC), from the scar to the tricuspid annulus.

Results Fifteen IARTs was induced in all of four patients. Four of 15 atrial tachycardias (ATs) were intra-scar reentry AT, three typical atrial flutter (AFL), two double loop reentry tachycardia (DLR) around superior vena cava (SVC) and inferior vena cava (IVC) respectively, four upper loop reentry tachycardia (ULR) around SVC, two lower loop reentry tachycardia (LLR) around IVC. The reentry loops of all ATs were related with scar area. The mean tachycardia cycle length was $268.1 \pm 49.5 \mathrm{~ms}$. In all patients, linear ablations along cavotricuspid isthmus, from scar area to crista terminalis and to IVC, were performed. Linear ablation from scar area to tricuspid annulus was performed in one patient. Isolation of scar area from 
right lateral wall was achieved in all patients. All tachycardias, including scar-related AT and typical AFL were ablated successfully. There was no complication during procedure. No recurrences of AT was observed during follow-up.

Conclusion Under conventional electrophysiological mapping, linear ablations from scar area to anatomic barrier and/or isolation of scar area from right lateral wall, could be successfully abolish IARTs and all potential circuits with reentry around or related to the scar.

\section{e0714 AUTONOMIC NERVE REMODELLING OF PROLONGED RAPID ATRIAL PACING-INDUCED ATRIAL FIBRILLATION AND REVERSAL EFFECT OF PROPRANOLOL}

doi:10.1136/hrt.2010.208967.714

Yang Yu, Li Liu, Jiu Yang Jiang. The First Hospital of Harbin Medical University

Background Limited study disclosed atrial heterogeneous sympathetic innervation in atrial fibrillation recently. But the changes of vagal nerve and the effect of medicine on neural remodelling are not clear.

Methods and Results Twelve dogs underwent atrial pacing at $600 \mathrm{bpm}$. Six of them were given propranolol (1 mg/ $\mathrm{kg}$ tid po, propranolol group), while the other six took placebo only. (AF group) All paced dogs developed sustained AF by 5 weeks of pacing. Tissues from six healthy dogs were used as controls. Immunohistochemistry staining of cardiac nerves was performed using anti-tyrosine hydroxylase $(\mathrm{TH})$ and anti-acetylcholine (Ach) antibodies. In AF group, the density of TH-positive in the right atrium (RA), atrial septum (AS) and left atrium (LA) was $2156.66 \pm 677.57 \mu \mathrm{m}^{2}$ per $\mathrm{mm}^{2}$, $3679.92 \pm 1951.41$ and $18961.8 \pm 10274.15 \mu \mathrm{m}^{2}$ per $\mathrm{mm}^{2}$ respectively, which was significantly $(p<0.01)$ higher than the nerve density in control tissues $(608.8 \pm 414.60,660.62 \pm 273.29$ and $792.46 \pm 326.33 \mu \mathrm{m}^{2}$ per $\mathrm{mm}^{2}$, respectively). In propranolol group, the density of TH-positive nerve in RA, AS, LA was $879.96 \pm 755.13 \mu \mathrm{m}^{2}$ per $\mathrm{mm}^{2}, 960.48 \pm 919.92$ and $1151.98 \pm 678.43 \mu \mathrm{m}^{2}$ per $\mathrm{mm}^{2}$ respectively, which was lower than the AF group. $(p<0.01)$ In AF group, the density of ACh-positive nerve in the RA, AS and LA was $721.94 \pm 341.07 \mu \mathrm{m}^{2}$ per $\mathrm{mm}^{2}, 279.86 \pm 110.25$ and $109.22 \pm 29.86 \mu \mathrm{m}^{2}$ per $\mathrm{mm}^{2}$ respectively, which was significantly $(\mathrm{p}<0.001)$ lower than the nerve density in control tissues (7102.32 \pm 2058.67 , $6093.20 \pm 2512.27$, and $5285.54 \pm 1982.96 \mu \mathrm{m}^{2}$ per $\mathrm{mm}^{2}$, respectively). In propranolol group, the density of ACh-positive nerve in RA, AS, LA was $5356.86 \pm 1733.25 \mu^{2}$ per $\mathrm{mm}^{2}, \quad 4774.86 \pm 1069.30$ and $3550.76 \pm 1399.55 \mu \mathrm{m}^{2}$ per $\mathrm{mm}^{2}$ respectively, which was higher than the AF group. $(p<0.01)$ It proved the truth that higher density of $\mathrm{TH}$ positive nerve $\left(10899.84 \pm 6365.15 \mu^{2}\right.$ per $\left.\mathrm{mm}^{2}, \mathrm{p}<0.001\right)$ and lower density of Ach-positive nerve $\left(127.94 \pm 88.98 \mu \mathrm{m}^{2}\right.$ per $\mathrm{mm}^{2}, \mathrm{P} 2 / \mathrm{mm}^{2}$ $(p<0.01)$ and $4223.22 \pm 1188.23 \mu^{2} / \mathrm{mm}^{2}$ respectively $(p<0.01)$.

Conclusion Sympathetic hyperinnervation and vagal denervation are associated with canine model of sustained AF produced by prolonged left atrial pacing. Propranolol can prevent autonomic remodel to some extent.

\section{e0715 LEFT ANTERIOR DESCENDING ARTERY DISSECTION AND ACUTE MYOCARDIAL INFARCTION AFTER BLUNT CHEST TRAUMA: TWO CASE REPORT}

doi:10.1136/hrt.2010.208967.715

${ }^{1}$ Longbin Liu, ${ }^{1}$ Hangyuan Guo, ${ }^{2}$ Haitao Lv, ${ }^{1}$ Yangbo Xin, ${ }^{1}$ Yufang Qiu, ${ }^{1}$ Fang Pen, ${ }^{1}$ Bio Yang. ${ }^{1}$ Department of Cardiology, Shaoxing People's Hospital, The First Affiliated Hospital of Shaoxing University, Shaoxing City, Zhejiang, China; ${ }^{2}$ Wenzhou Medical College, Wenzhou City, Zhejiang, China

Blunt chest trauma can cause several forms of cardiac injury. Of these, coronary artery dissection and acute myocardial infarction is a rare complication. We report two cases of the left anterior descending coronary artery dissection and acute myocardial infarction after blunt chest trauma.

\section{e0716 AN 81-YEAR-OLD MAN WITH DIZZINESS, FATIGUE AND SHORTNESS OF BREATH}

doi:10.1136/hrt.2010.208967.716

Nan Li. Honorary Clinical Research Associate, Department of Medicine, University of Hong Kong

Case An 81-year-old man presented with dizziness, fatigue and shortness of breath one day after a min-invasive neck spine surgery. The patient said the surgery had been successful, and he was able to get up and walk on the first day, but on the second day he felt dizzy, tired, and dyspnoea. He also found his two hands were swelling. He denied chest pain, unconsciousness, vomiting and other symptoms. The patient was on medication of hypertension and diabetes before the surgery, and both of his wife and son have hypertension. The patient does not smoke nor drink. After surgery, the patient was given antibiotics by venous infusion. On physical examination, the patient looked conscious, well nourished but having a big belly. His temperature was normal. His four extremities were swelling. He had jugular retention. His blood pressure was 164/83 mm Hg, heart rate was $86 \mathrm{bpm}$ and respiratory rate was 24 . Heart auscultation revealed no heart murmur and normal heart rhythm. Pulmonary auscultation found some moist rales at the lower part of two lungs. Chest $\mathrm{x}$-ray showed his heart was boot-shaped, lungs were clear and there was a little pleural effusion in the thorax. Cervical vertebra x-ray showed that the third and fourth spine cords were fixed by steel and there was ossification of nuchal ligament. ECG was almost normal. Echocardiography showed that the internal diameter of left atrium was $50 \mathrm{~mm}$ at diastolic phase; the right atrium was enlarged and the left ventricular posterior wall a bit thicker; the aortic sinus and proximal ascending aorta were widened; the left ventricular ejection fraction was $66 \%$; the diastolic function was slightly reduced. The complete blood count showed: white-cell count $6.11 \times 10^{9} / 1$ neutrophil-cell count $4.22 \times 10^{9} / 1$, percentage of neutrophil-cell $69.1 \%$, red-cell count $4.24 \times 10^{12}$, haemoglobin $125.0 \mathrm{~g} / \mathrm{l}$, haematocrit $37.6 \%$, platelet count $136 \times 10^{9} / 1$. The biochemistry test showed: serum potassium $4.1 \mathrm{mmol} / 1$, serum sodium $142.0 \mathrm{mmol} / \mathrm{l}$, serum chloride $99 \mathrm{mmol} / \mathrm{l}$, carbon dioxide $24.0 \mathrm{mmol} / \mathrm{l}$, serum calcium $2.53 \mathrm{mmol} / \mathrm{l}$, creatinine $124.0 \mathrm{ummol} / \mathrm{l}$, blood urea nitrogen $8.2 \mathrm{mmol} / \mathrm{l}$, uric acid $527.0 \mathrm{umol} / 1$. Serum brain natriuretic peptide level: $348 \mathrm{pg} / \mathrm{ml}$. Blood glucose level: $6.88 \mathrm{mmol} / \mathrm{l}$. The diagnosis of this patient was based on his symptoms of fatigue, and shortness of breath at rest; physical examination of jugular retention, lung rales at lower part and ankle oedema; chest x-ray of pleural effusion. These symptoms and findings indicated the patient had pulmonary congestion and systemic congestion. Though ECG was normal, echocardiography showed diastolic dysfunction, and the serum BNP level was significantly raised. Thus the patient was diagnosed as: acute heart failure, diastolic heart failure, NYHA IV, Stage C; hypertension, grade 3; type 2 diabetes mellitus; cervical spondylopathy, post mini-invasive spine surgery. The treatment included blood pressure monitoring, urinary volume monitoring and electrocardiographic monitoring. Medications included vasodilator nitrate to relief cardiac workload; loop diuretic furosemide to induce diuresis; ACE inhibitor fosinopril to control hypertension; statin atorvastatin to lower lipid level; Insulin to control glucose level. The patient stayed in hospital for 10 days and discharged with no dyspnoea and low extremity oedema, no pulmonary rales, blood pressure $139 / 69 \mathrm{~mm} \mathrm{Hg}$ and heart rate $57 \mathrm{bpm}$. During the 3 months follow-up after discharge, the patient strictly followed doctor's written instructions on diet restriction and exercise. $\mathrm{He}$ measured blood pressure, heart rate and weight and then recorded 